\title{
ПРИМЕНЕНИЕ МЕСТНОЙ АНЕСТЕЗИИ ПРИ ВИДЕОТОРАКОСКОПИЧЕСКОЙ РЕЗЕКЦИИ ЛЕГКОГО
}

\author{
П. П. Шипулин, Е. Ю. Тронина, А. А. Кирилюк, В. В. Байдан, В. Е. Севергин, \\ О. Н. Козяр, А. Аграхари
}

Одесская областная клиническая больница

\section{APPLICATION OF LOCAL ANESTHESIA WHILE PERFORMANCE OF VIDEOTHORACOSCOPIC PULMONARY RESECTION}

\author{
P. P. Shipulin, E. Yu. Tronina, A. A. Kyrylyuk, V. V. Baydan, V. E. Severgin, O. N. Kozyar, A. Agrahari \\ Odessa Regional Clinical Hospital
}

\section{Реферат}

Цель. Анализ результатов использования местной анестезии ((МА) при выполнении видеоторакоскопической резекции легкого (ВТРЛ) у пациентов пожилого возраста при тяжелых сопутствующих соматических заболеваниях.

Материалы и методы. Представлен опыт выполнения ВТРЛ с использованием МА у 61 пациента по поводу очаговых и диссеминированных заболеваний легких. Показана возможность выполнения операции под МА с применением эндостаплеров (ЭС) и электросварочных инструментов.

Результаты. Все больные благополучно перенесли миниинвазивные операции с использованием МА, отмечено уменьшение длительности операции и лечения в стационаре до 9,4 дня. Осложнение в виде временной негерметичности легкого возникло у $6(9,8 \%)$ больных.

Выводы. ВТРЛ под МА хорошо переносится больными, что позволяет выполнять ее как с диагностической, так и лечебной целью, что особенно важно у пациентов пожилого возраста и при наличии тяжелых сопутствующих заболеваний.

Ключевые слова: видеоторакоскопическая резекция легкого; местная анестезия; электросварочная резекция легкого; эндостаплеры.

Abstract

Objective. Analysis of results of the local anesthesia application while doing videothoracoscopic pulmonary resection (VTHPR) in elderly patients, suffering severe somatic concurrent diseases.

Materials and methods. Experience of VTHPR performance, using local anesthesia in 61 patients, suffering focal and disseminated pulmonary diseases, was presented. Possibility of the operation performance under local anesthesia, using endostaplers and electric welding instruments, was analyzed.

Results. All the patients have survived miniinvasive operations, using local anesthesia, the operative intervention and the stationary treatment duration down to 9.4 days was noted. Complications of temporal pulmonary leakage have occurred in 6 (9.8\%) patients. Conclusion. VTHPR under local anesthesia is well tolerated by the patients, what permits its application for diagnostic and treatment purposes with great gain for aged patients, suffering concurrent severe diseases.

Keywords: videothoracoscopic pulmonary resection; local anesthesia; electric welding pulmonary resection; endostaplers.

Общепринятое анестезиологическое обеспечение ВТРЛ в виде общего обезболивания с обязательным выключением из дыхания оперируемого легкого не всегда возможно у пациентов пожилого возраста при тяжелых сопутствующих заболеваниях. Высок риск подобных вмешательств у пациентов при хронических обструктивных заболеваниях легких (ХОЗЛ), когда выключение из дыхания оперируемого легкого не представляется возможным. Эти обстоятельства обусловили необходимость разработки миниинвазивных торакоскопических операций, включая лобэктомию, с использова- нием МА, без интубации трахеи [15].

Цель исследования: анализ результатов использования МА при выполнении ВТРЛ.

\section{МАТЕРИАЛЫ И МЕТОДЫ ИССЛЕДОВАНИЯ}

МА применена при выполнении ВТРЛ у 61 больного в возрасте от 16 до 75 лет, преимущественно пожилого возраста, мужчин - 41, женщин -20 .

Хронические неспецифические и нагноительные заболевания легких и плевры диагностированы у 48 пациентов, первичные и метастатиче- ские злокачественные опухоли легких - у 9, туберкулезное поражение легких - у 3, фиброзирующий альвеолит - у 1. У большинства пациентов выявлены сопутствующие заболевания и дыхательная недостаточность.

Поражение легких было многоочаговым либо диссеминированным, неясной этиологии, у 24 пациентов - сочеталось с плевральным выпотом в виде серозного либо гнойного экссудата.

Показаниями к использованию МА были преклонный возраст, тяжелые сопутствующие заболевания с признаками дыхательной недостаточности, отказ больных от об- 
щего обезболивания. У некоторых больных при выполнении видеоторакоскопической операции по поводу экссудативного плеврита или острой эмпиемы плевры под МА обнаруженные во время вмешательства патологические изменения легкого потребовали выполнения дополнительной ВТРЛ.

Все оперативные вмешательства выполняли с использованием бокового доступа при спонтанном дыхании пациента.

При ВТРЛ использовали только закрытую методику операции через 3 торакопорта, без миниторакотомного доступа.

Методика МА предполагала последовательную анестезию точек установки троакаров раствором анестетика (2,5\% лидокаин либо 0,5\% новокаин) и существенно не отличалась От МА, применяемой при диагностической торакоскопии. При необходимости дополнительной биопсии париетальной плевры осуществляли анестезию соответствующего участка. Зону введения троакаров определяли в зависимости от предполагаемой локализации патологического очага, выбирали индивидуально. При этом троакары обязательно располагали в виде треугольника во избежание эффекта фехтования эндоскопическими инструментами.

Перед внутриплевральными манипуляциями детально осматривали плевральную полость, при наличии экссудата его обязательно удаляли.

Все ВТРЛ были краевыми либо атипичными. Применяли ЭС «Endopath Echelon» EC 45 и 60 с прямой и вращающейся рабочей частью и «Covidien Endo GIA-60», а также электросварочные хирургические комплексы «ЕК-300 M1», «Liga Sure Valleylab, «УХВЧ-150-Фотек» с соответствующим набором эндоскопических инструментов. У некоторых больных сочетали ЭС с электрохирургической техникой. Методика ВТРЛ с применением МА существенно не отличалась от ВТРЛ под общим обезболиванием.

\section{РЕЗУЛЬТАТЫ}

Все пациенты благополучно перенесли оперативное вмешательство и выписаны.
Объем ВТРЛ под МА не превышал краевую и атипичную резекцию. У 37 (60,6\%) больных ВТРЛ выполнена с помощью ЭС и механического шва, у 21 (34,4\%) - использовали электросварочный бесшовный метод резекции, у 3 (5,0\%) - сочетание механического и электросварочного швов.

Технически выполнение атипичной резекции с помощью ЭС не отличалось от общепринятых методик. Наличие спонтанного дыхания не представляло проблем при выполнении вмешательства. Для извлечения резецированного препарата несколько расширяли рану одного из троакаров. Все пациенты благополучно перенесли ЭС ВТРЛ под МА. Электросварочную резекцию обычно выполняли с иссечением небольших участков легкого, которые направляли для морфологического исследования. Линия сварочного шва при краевой и атипичной резекции была герметичной, не требовала дополнительного укрепления. Возникший у 6 (9,8\%) больных сброс воздуха был кратковременным, устранен путем активной аспирации из плевральной полости в течение нескольких дней.

Отмечено уменышение длительности операции в среднем на 45 мин. Также наблюдали уменьшение выраженности болевого синдрома, что не требовало введения наркотических препаратов. В среднем продолжительность лечения больного в стационаре составил 9,4 дня. Осложнения в виде остаточной полости возникли у 7 (11,5\%) больных.

\section{ОБСУЖДЕНИЕ}

K. Nezu и соавторы [1], одними из первых начавшие широкое использование МА при ВТРЛ, показали безопасность подобных вмешательств и значительное уменьшение продолжительности лечения больных в стационаре. Это подтверждено и в более поздних исследованиях [2, 4-6]. Отмечено значительное уменьшение частоты послеоперационных осложнений при использовании МА $[7,8]$. У пациентов при наличии сопутствующих ХОЗЛ преимущества MA превышают недостатки этого метода [7]. При проведении однолегочной вентиляции во время ВТРЛ авторы отметили отсутствие воспалительной реакции организма на этот метод, что еще раз подтвердило преимущества МА у пожилых и ослабленных пациентов [9].

Непременным условием при проведении ВТРЛ с использованием МА считают, помимо анестезии межреберных нервов, общую седацию пациента [10]. В наших наблюдениях все ВТРЛ были успешно выполнены только при анестезии зоны введения троакаров. Постепенно возникающий коллапс легкого при использовании спонтанного дыхания позволил выполнить его атипичную резекцию с использованием как ЭС, так и электросварочной техники.

МА является эффективным и безопасным способом при выполнении торакоскопических операций по поводу неопухолевых и опухолевых заболеваний легких, в том числе злокачественного плеврита, эмпиемы плевры, спонтанного пневмоторакса, интерстициального поражения легких [4]. Некоторые авторы не применяли наркоз при выполнении видеоторакоскопической краевой резекции легкого по поводу буллезной эмфиземы [8]. С успехом использовали МА при проведении объем-редуцирующих операций по поводу эмфиземы легких [6].

Дальнейшее совершенствование техники миниинвазивных вмешательств на легких позволило применить МА даже при таких сложных операциях, как лобэктомия [3, 5]. Применение ВТРЛ для выполнения такого оперативного объема оправдано только у пациентов пожилого возраста при высоком риске осложнений [5]. Во время выполнения операции возможно возникновение кашля, что не позволяет осуществить лимфодиссекцию и работать в зоне главных бронхов.

Наложение механического и электросварочного швов использовали при выполнении большой по объему резекции, что позволяло экономить число картриджей ЭС.

Основным показанием к проведению ВТРЛ под МА была необходимость морфологической верификации патологического процесса. Эта задача выполнена у всех пациентов. При наличии плеврального выпота 
неясного генеза только видеоторакоскопическая биопсия плевры не всегда позволяет уточнить характер патологического процесса, что требует резекции небольших участков ткани легких. Использование электросварочных методик при этом достаточно для выполнения атипичной резекции по типу биопсии легкого и не требует применения дорогостоящих ЭС.

При выполнении миниинвазивных вмешательств по поводу острой эмпиемы плевры предварительно осуществляли видеоторакоскопическую декортикацию легкого с объединением отдельных гнойных полостей в единую. Показания к ВТРЛ в такой ситуации возникают при предположении о специфической этиологии гнойного процесса (туберкулез, метастатическое поражение). В таких ситуациях краевую резекцию небольших участков легкого используют для последующего морфологического исследования. При этом возможно не только гистологическое, но и микробиологическое

\section{REFERENCES}

1. Nezu K, Kushibe K, Tojo T, Takahama M, Kitamura S. Thoracoscopic wedge resection of blebs under local anesthesia with sedation for treatment of a spontaneous pneumothorax. Chest. 1997;111:230-5.

2. Deng HY, Zhu ZJ, Wang YC, Wang WP, Ni PZ, Chen LQ. Non-intubated video-assisted thoracoscopic surgery under loco-regional anesthesia for thoracic surgery: a meta-analysis. Interact Cardiovasc Thorac Surg. [Internet]. 2016 Mar. [cited 2016 Jul 1];23:31-40. doi: 10.1093/icvts/ivw055. Available from: https://academic.oup. com/icvts/article-lookup/doi/10.1093/icvts/ivw055.

3. Gonzalez-Rivas D, Fernandez R, de la Torre M, Rodriguez JL, Fontan L, Molina F. Single-port thoracoscopic lobectomy in a nonintubated patient: the least invasive procedure for major lung resection? Ibid. 2014. Jul [cited 2014 Oct 1];19(4):552-5. doi: 10.1093/icvts/ ivu209. Available from: https://academic.oup.com/icvts/article-lookup/doi/10.1093/icvts/ivu209.

4. Mineo TC, Tacconi F. From "awake" to "monitored anesthesia care" thoracic surgery: a 15 year evolution. Thorac Cancer. [Internet]. 2014 Jan [cited 2014 Jan 2];5(1):1-13. doi: 10.1111/1759-7714.12070. Available from: https://jnlinelibrary.wiley.com/doi/10.1111/1759_ 7714.12070/full.

5. Roco G. Non-intubated lung surgery. Eur J Cardiothorac Surg. [Internet]. 2016 Jan [cited 2016 Apr 8];49 Suppl. 1:13-5. doi: 10.1093/ ijcts/ezv412. Available from: https://academic.oup.com/ijcts/articlelookup/doi/10.1093/ijcts/ezv412.

6. Pompeo E, Tacconi F, Mineo TC. Comparative results of non-resectional lung volume reduction performed by awake or non-awake anesthesia. Ibid. [Internet]. 2011 Apr;39(4):51-8. doi: 10.1016/j. ejcts.2010.11.071. Available from: https://academic.oup.com/ejcts/ article-lookup/doi/10.1016/j.ejcts.2010.11.071. лечения больного в стационаре, хорошей переносимости вмешательства, уменьшения частоты послеоперационных осложнений [2].

\section{выводы}

1. МА позволяет выполнять ВТРЛ как с диагностической, так и лечебной целью, что особенно важно у пациентов пожилого возраста и при наличии тяжелых сопутствующих заболеваний.

2. Проведение ВТРЛ под МА с использованием электросварочных методик существенно не отличается от подобных операций под общим обезболиванием.

3. Важными преимуществами ВТРЛ под МА являются хорошая переносимость, даже больными пожилого возраста, отсутствие тяжелых осложнений и летального исхода, уменьшение длительности лечения больного в стационаре.

4. Необходимо уточнение показаний к применению метода в клинической практике.

7. Hausman MSJr, Jewell ES, Engoren M. Regional versus general anesthesia in surgical patients with chronic obstructive pulmonary disease: does avoiding general anesthesia reduce the risk of postoperative complications? Anesth Analg. [Internet]. 2015 Jun;120(6):140512. doi: 10.1013/ANE.0000000000000574. Available from: https:// journals.Iww.com/anesthesia-analgesia/Fulltext/2015/06000/Regional_Versus_General_Anesthesia_in_Surgical.32.aspx

8. Liu J, Cui F, Li S, Chen H, Shao W', Liang L, et al. Nonintubated video-assisted thoracoscopic surgery under epidural anesthesia compared with conventional anesthesia option: a randomized control study. Surg Innov. [Internet]. 2014 May 12 [cited 2015 Apr ];22(2):123-390. doi: 10.1177/1553350614531662. Available from: https://journals.sagepub.com/doi/10.1177/1553350614531662.

9. Sagasawa Y, Yamaguchi K, Kumakura S, Murakami T, Kugimiya $\mathrm{T}$, Suzuki K, et al. The effect of one-lung ventilation upon inflammatory responses during lung resection. J Anesth. [Internet]. 2011 Feb 8; [cited 2011 Apr];25(2):170-7. doi: 10.1007/s00540-0111100-0. Available from: https://link.springer.com/article/10.1007\%2 Fs00540-011-1100-0.

10. Hung MH, Hsu HH, Chan KC, Chen KC, Yie JC, Cheng YJ, et al. Non-intubated thoracoscopic surgery using internal intercostal nerve block, vagal block and targeted sedation. Eur J Cardiothorac Surg. [Internet]. 2014 Feb 28;[cited 2014 Oct];46(4):620-5. doi: 10.1093/ijcts/ezu054. Available from: https://academic.oup.com/ ejcts/article-lookup/doi/10.1093/ejcts/ezu054.

11. Djouzno XB. Lung biopsies for interstitial lung disease: the limits of traditional methods of microbiological identification. Ibid. [Internet]. 2012 Jan 1[cited 2012 Mar];41(1):39-40. doi: 10.1016/j. ejcts/2011.04.009. Available from: https://academic.oup.com/ejcts/ article-lookup/doi/10.1016/j.ejcts.2011.04.009. 